\title{
The Massive Close Binary in the $\delta$ Ori A Triple System
}

\author{
James A. Harvin \& Douglas R. Gies \\ Center for High Angular Resolution Astronomy, Department of Physics \\ and Astronomy, Georgia State University, Atlanta, GA 30303, USA
}

\begin{abstract}
We present an analysis of short-wave, high-dispersion ultraviolet spectra of the triple star $\delta$ Ori A from the International Ultraviolet Explorer Satellite's (IUE) Final Archive. These spectra were cross-correlated against $\mathrm{AE}$ Aur to find the components' radial velocities, which were then used to produce the system's orbital elements. The long-period tertiary star in the $\delta$ Ori A system was not seen in the resulting cross-correlation functions (CCFs). The close binary's eclipses allow the orbit's inclination to be estimated by modeling of its Hipparcos light curve. The primary star appears to have a mass of $11.2 \mathrm{M}_{\odot}$ and the secondary seems to have a mass of $5.6 \mathrm{M}_{\odot}$, both of which are about $1 / 3$ of the expected values for stars of their MK types. Although we expected the massive close binary in the $\delta$ Ori A system to be a pre-Roche lobe overflow (RLOF) system, these masses appear to require that it be a post-RLOF system. The full description of this work, including the tomographic separation of the spectra for the close binary's components, appears in Harvin et al. (2002).
\end{abstract}

\section{Introduction}

$\delta$ Ori (Mintaka, 34 Ori, HD 36486, HIP 25930), the westernmost of Orion's Belt Stars, is a multiple star system with a total of five visual and spectroscopic components as depicted in Fig. 1. The visual primary, $\delta$ Ori Aa, has three companions in the Washington Double Star (WDS) Catalog (Worley \& Douglass 1997). Two of the visual companions, $\delta$ Ori B (BD-00 983B), a 14.0 mag star $33^{\prime \prime}$ away reported by S. W. Burnham, and $\delta$ Ori C (HD 36485), a $6.85 \mathrm{mag}$ star $53^{\prime \prime}$ away reported by F. G. W. Struve, have been known for more than a hundred years. Neither of them is close enough to $\delta$ Ori A to contaminate its $I U E$ spectra. $\delta$ Ori A, itself, is composed of $\delta$ Ori Aa, a single-lined spectroscopic binary with an O9.5 II primary (Walborn 1972) and a period of 5.7325 (Harvey et al. 1987), and the third WDS visual companion, $\delta$ Ori Ab (Heintz 1980). According to the Hipparcos Catalog (Perryman 1997), $\delta$ Ori Ab is $1.4 \mathrm{mag}$ fainter than $\delta$ Ori Aa, and the separation between them is only a few tenths of an arcsecond. Therefore, the $I U E$ spectra of $\delta$ Ori A should be a composite of the contributions of both components of the spectroscopic binary and $\delta$ Ori Ab.

Single-lined orbital solutions for the spectroscopic binary span the last century (Harvey et al. 1987), and because it contains an eclipsing binary, $\delta$ Ori A has also been the subject of several photometric studies (Koch \& Hrivnak 1981). 


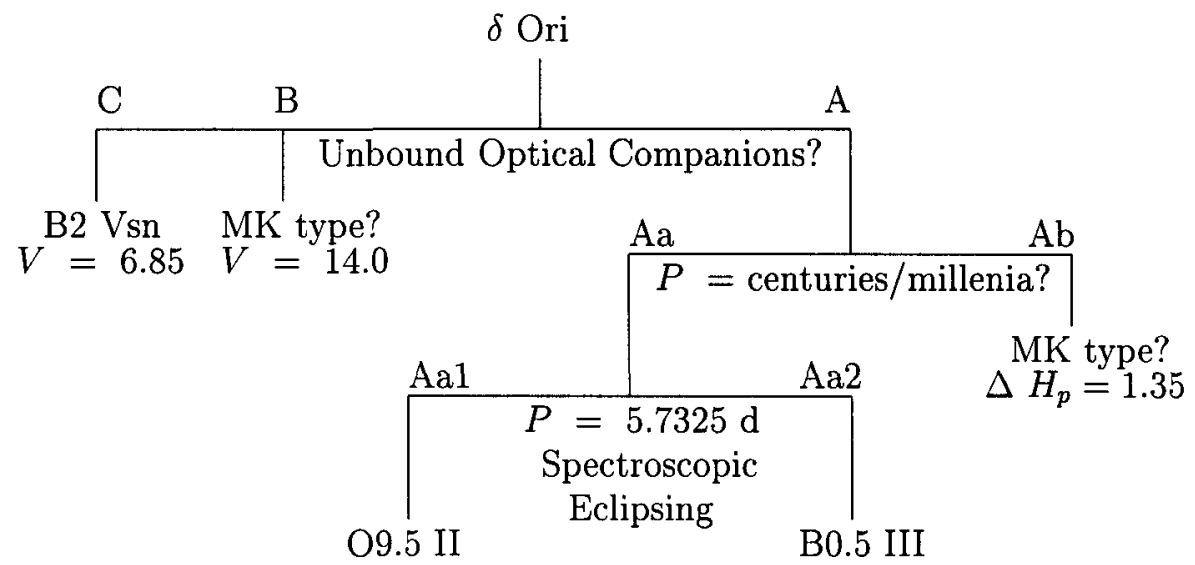

Figure 1. The Visual and Spectroscopic Components of $\delta$ Ori

We believe we have now located the secondary's spectroscopic signature, but our measured velocity amplitude leads to masses that certainly make the components of $\delta$ Ori Aa two of the most exotic stars in the entire sky.

\section{IUE Observations}

There are 60 short-wave, high-dispersion spectra of $\delta$ Ori A available in the IUE Final Archive at the Space Telescope Science Institute's Multi-mission Archive'. The spectra were retrieved in NEWSIPS format (Garhart et al. 1997) and processed using local procedures written in IDL $^{2}$. These spectra, made with both the large and small apertures, have a signal-to-noise ratio $(\mathrm{S} / \mathrm{N})$ of approximately 10 per pixel. The spectra were then cross-correlated with the spectrum of a narrow-lined reference star, AE Aur (HD 34078), which has the properties: $09.5 \mathrm{~V}, V \sin i=30 \mathrm{~km} \mathrm{~s}^{-1}$, and $V_{r}=+54.4 \mathrm{~km} \mathrm{~s}^{-1}$ (Gies \& Bolton 1986). The regions around the broad features of Ly $\alpha$, Si IV $\lambda 1400$, and CIV $\lambda 1550$ were excluded from the CCFs.

In order to find the most accurate shift positions (and therefore, radial velocities) for each of the components in the composite CCFs, we simultaneously fitted two profiles, one for each of the binary's components, to each individual CCF using a non-linear, least-squares fit. The model for the primary star's CCF was created by summing the short wavelength half of the composite profile from the blue-shifted quadrature phases of the primary's orbit with the long wavelength half of the composite profile from the red-shifted quadrature phases using a windowing function. We used a Gaussian as the functional shape for the secondary's CCF component. No signal from the tertiary's spectrum was detected in the CCF residuals during this process, and we estimate that the presence of a

\footnotetext{
${ }^{1}$ Available at http://archive.stsci.edu/iue.

${ }^{2}$ IDL is a registered trademark of Research Systems, Inc.
} 


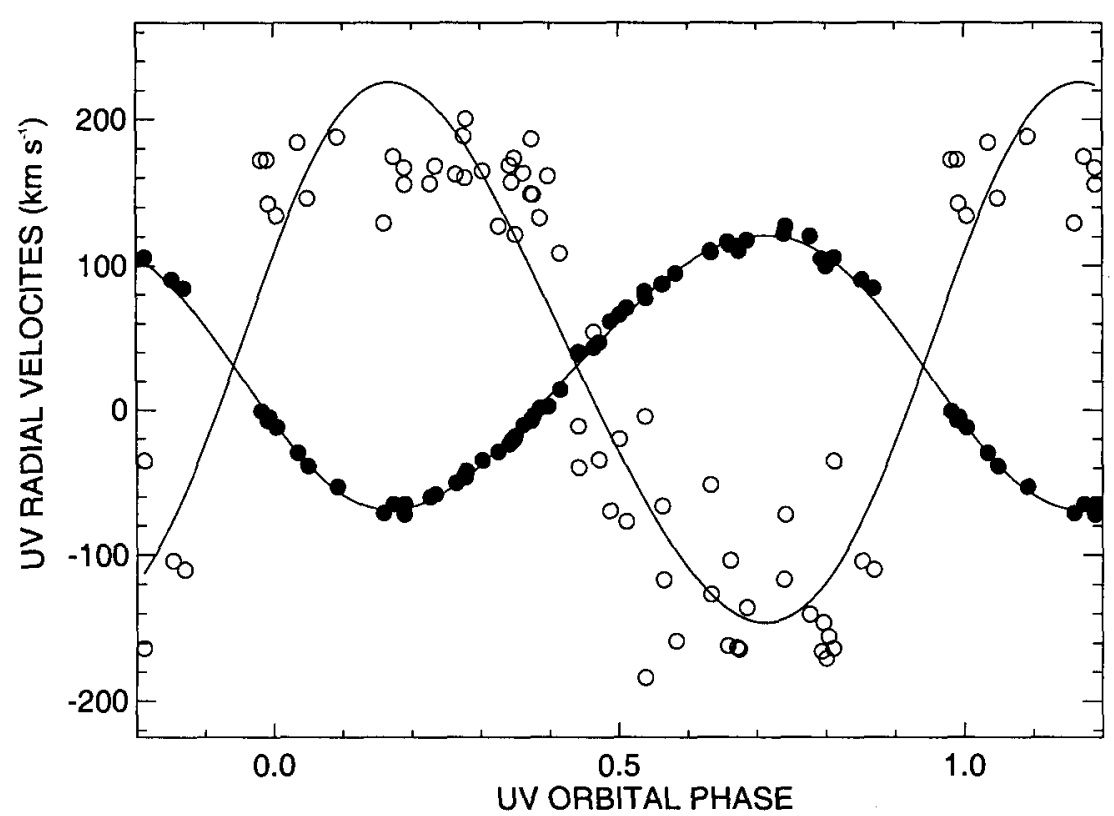

Figure 2. The Double-lined Orbit from IUE Radial Velocities. Filled circles: primary star velocities. Open circles: secondary star velocities.

signal from an undetected tertiary spectrum similar to the secondary's spectrum in the CCFs could raise our estimated masses by, at most, $43 \%$. The absence of the tertiary's spectrum in the CCFs (it contributes 30\% of the system's total flux in $V$ ) could be explained if it is a very rapid rotator or a spectroscopic binary.

\section{Radial Velocities \& Orbital Elements}

The radial velocities from our simultaneous fitting procedure are plotted in Fig. 2. We found the following system orbital elements using the non-linear, least-squares fitting program of Morbey \& Brosterhus (1974):

- $P=5.732503 \pm 0.000026 \mathrm{~d}$,

- $K_{1}=94.9 \pm 0.6 \mathrm{~km} \mathrm{~s}^{-1}, K_{2}=186 \pm 9 \mathrm{~km} \mathrm{~s}^{-1}$,

- $e=0.075 \pm 0.06$.

The deviation of the secondary star's radial velocities at the receding quadrature remains unexplained. These elements were then used to calculate the following masses for the two components of the close binary, $\delta$ Ori Aa1 and $\delta$ Ori Aa2:

- $M_{1} \sin ^{3} i=8.7 \pm 1.4 \mathrm{M}_{\odot}$,

- $M_{2} \sin ^{3} i=4.4 \pm 0.3 \mathrm{M}_{\odot}$. 


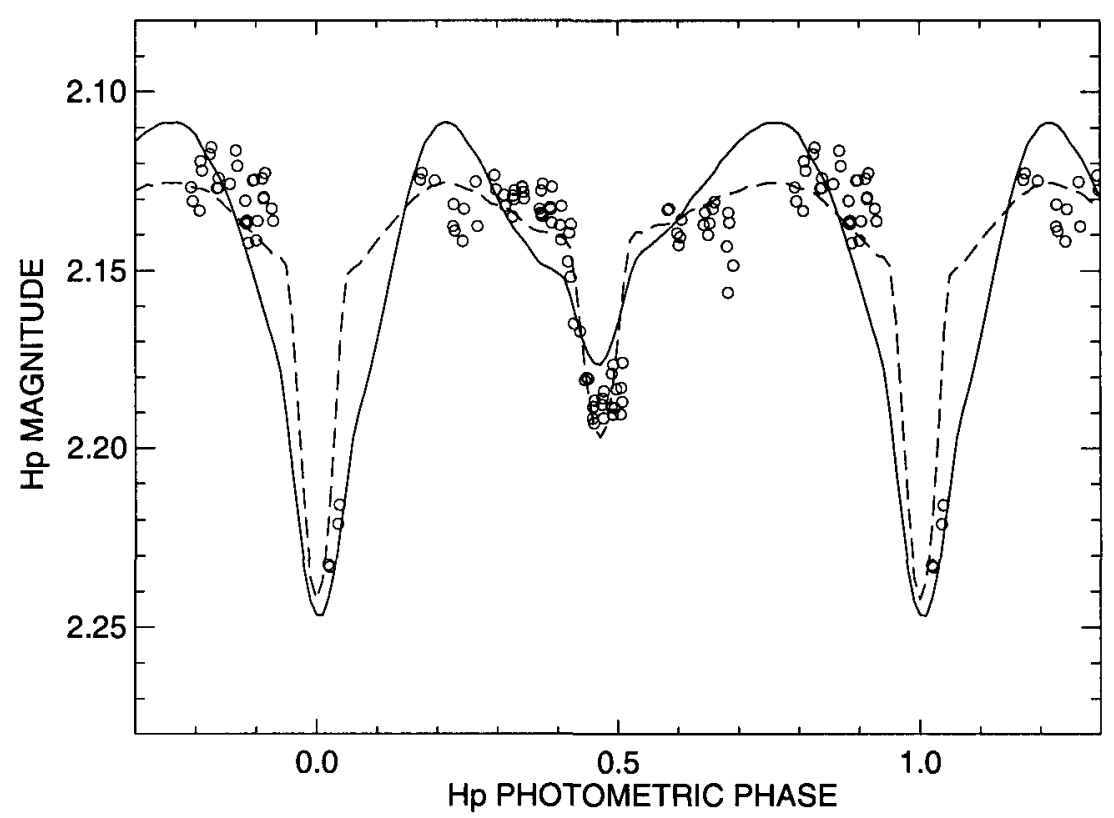

Figure 3. Hipparcos Photometric Data. Solid line: $i=67^{\circ}$ and $R_{p}=13 \mathrm{R}_{\odot}$. Dashed line: $i=77^{\circ}$ and $R_{p}=9 \mathrm{R}_{\odot}$.

\section{Hipparcos Photometry}

The Hipparcos Catalog contains photometric data from each object that Hipparcos observed in its Hipparcos Epoch Photometry Annex ${ }^{3}$. The light curve fitting was done with two free parameters, the system's orbital inclination and the primary star's radius. The secondary star's radius was fixed at $R_{s} / R_{p}=0.37$ based on the recovered flux ratio from Doppler tomography (Harvin et al. 2002) and its expected MK type. Given the primary star's effective temperature, $T_{\text {eff }}=33,000 \mathrm{~K}$ (Voels et al. 1989), we were able to base our subsequent analysis on an inclination of $i=67^{\circ}$. At $i=67^{\circ}$, the primary star radius is $13 \mathrm{R}_{\odot}$, which gives a luminosity that is consistent with the closer photometric distance estimates of $360 \pm 70 \mathrm{pc}$ for $\delta$ Ori A (Brown, de Geus, \& de Zeeuw 1994) and exactly one standard deviation behind its Hipparcos parallax. The physical properties of the close binary's primary and secondary stars, $\delta$ Ori Aal and Aa2, are given in Table 1.

\footnotetext{
${ }^{3} \mathrm{http}: / /$ astro.estec.esa.nl/Hipparcos/research.html
} 
Table 1. The Physical Properties Assuming $i=67^{\circ}$

\begin{tabular}{lcc}
\hline \multicolumn{1}{c}{ Category } & Primary & Secondary \\
\hline Sp. Type & O9.5 II & B0.5 III \\
$\Delta m(1600 \AA)$ & $\ldots$ & 2.6 \\
$V \sin i\left(\mathrm{~km} \mathrm{~s}^{-1}\right)$ & $157 \pm 6$ & $138 \pm 16$ \\
Radius $\left(R / R_{\odot}\right)$ & 13 & 5 \\
Mass $\left(M / M_{\odot}\right)$ & $11.2 \pm 1.8$ & $5.6 \pm 0.4$ \\
\hline \hline
\end{tabular}

\section{The Evolutionary Status of the $\delta$ Ori Aa System}

$\delta$ Ori Aa was expected to be a pre-RLOF object, but the masses in Table 1 are about $1 / 3$ of the expected values for the primary and secondary stars $(\approx 33$ and $18 \mathrm{M}_{\odot}$, respectively), indicating this is a highly evolved post-RLOF system. There is no evidence of large-scale gas streams in the system, so rapid-phase RLOF must be complete in any classical RLOF mass exchange scenario. Also, the orbit should have circularized during rapid-phase RLOF, but we found $e=$ 0.075 , consistent with most of the other orbits found over the last hundred years.

Because most stellar systems, no matter how strange, are likely to have evolved from Zero-Age Main Sequence (ZAMS) progenitors, we have considered three early Case A RLOF scenarios for producing a close binary like the one we propose for $\delta$ Ori Aa. Case A RLOF is RLOF in a binary system when its stars are still on the Main Sequence. Positing early Case A RLOF helps explain the lack of nuclear-processed abundances on the surface of the primary star.

\section{The primary was the donor star in quasi-conservative RLOF}

In this case, most of the overflowing mass must have been lost from the system, but a little mass was received by the secondary causing it to become overluminous. The problem is that the primary should display altered CNO abundances on its surface, but these are not seen in the $I U E$ spectra.

\section{The secondary was the donor star in quasi-conservative $R L O F$}

Some mass must have been received by the current primary causing it to become overluminous, but as in the case above, most of the overflowing mass must have been lost from the system. In this case, the problems are that the current secondary should be much hotter, and the current primary appears to be too overluminous.

\section{Both stars became donor stars by common envelope ejection}

In this case, the system underwent early Case A RLOF and formed a common envelope (CE). By an unknown mechanism, the CE was ejected (CEE) before a merger could occur. The conditions at the CE's surface should be typical of an OBA supergiant, except that the surface gravity would be further lowered by strong Coriolis forces due to the rapid "rotation" (at the system's orbital velocity) of the CE. A dramatically enhanced wind on the order of $10^{-3} \mathrm{M}_{\odot}$ $\mathrm{yr}^{-1}$ ) seems possible. 
Such a CEE event would require $\approx 35,000 \mathrm{y}$ to remove $\approx 35 \mathrm{M}_{\odot}$ of stellar material, which is about four times the $8,500 \mathrm{y}$ thermal timescale of an $\approx 51 \mathrm{M}_{\odot}$, $35 \mathrm{R}_{\odot}$ progenitor CE system "rotating" with a $2 \mathrm{~d}$ orbital period.

Since the CE undergoing ejection is bound to the system, but not to the individual stellar cores, the mass bound to the individual stellar cores (the mass inside of each component's Roche lobe, which is bound more tightly and subject to smaller Coriolis forces) would be left behind after the CEE event. This explains why neither of $\delta$ Ori Aa's components became a denuded stellar core.

The CEE scenario has several additional advantages over the pure RLOF scenarios. CEE can explain how both stars, not just the recipient star, became undermassive for their luminosities, why both stars appear to be underluminous for their MK types, why the primary star appears to have primordial rather than CNO-processed abundances on its surface, and finally, how this system could lose $2 / 3$ of its mass, which should have magnified the orbital period by a factor of about $10^{6}$, and still have an orbital period of only 5.73. Quasi-conservative RLOF scenarios will have great difficulty explaining these points.

\section{References}

Brown, A. G. A., de Geus, E. J., \& de Zeeuw, P. T. 1994, A\&A, 289, 101

Garhart, M. P., Smith, M. A., Turnrose, B. E., Levay, K. L., \& Thompson, R. W. 1997, International Ultraviolet Explorer New Spectral Image Processing System Information Manual, Version 2.0 (Greenbelt: NASA)

Gies, D. R., \& Bolton, C. T. 1986, ApJS, 61, 419

Harvey, A. S., Stickland, D. J., Howarth, I. D., \& Zuiderwijk, E. J. 1987, Observatory, 107, 205

Harvin, J. A., Gies, D. R., Bagnuolo, W. G., Penny, L. R., \& Thaller, M. L. 2002, ApJ, 565, 1216

Heintz, W. D. 1980, ApJS, 44, 111

Koch, R. H., \& Hrivnak, B. J. 1981, ApJ, 248, 249

Morbey, C. L., \& Brosterhus, E. B. 1974, PASP, 86, 455

Perryman, M. A. C. 1997, The Hipparcos and Tycho Catalogues (ESA SP-1200) (Noordwijk: ESA)

Voels, S. A., Bohannan, B., Abbott, D. C., \& Hummer, D. G. 1989, ApJ, 340, 1073

Walborn, N. R. 1972, AJ, 77, 312

Worley, C. E., \& Douglass, G. G. 1997, A\&AS, 125, 523 